\title{
Bimodal spectral variability of Cygnus X-1 in an intermediate state
}

\author{
J. Malzac ${ }^{1}$, P. O. Petrucci ${ }^{2}$, E. Jourdain ${ }^{1}$, M. Cadolle Bel ${ }^{3,4}$, P. Sizun ${ }^{3}$, G. Pooley ${ }^{5}$, C. Cabanac ${ }^{2}$, S. Chaty ${ }^{6}$, \\ T. Belloni ${ }^{7}$, J. Rodriguez ${ }^{3,6,8}$, J. P. Roques ${ }^{1}$, P. Durouchoux ${ }^{3}$, A. Goldwurm ${ }^{3,4}$, and P. Laurent ${ }^{3,4}$
}

\author{
${ }^{1}$ Centre d'Étude Spatiale des Rayonnements (CNRS/UPS/OMP), 31028 Toulouse, France \\ e-mail: Julien.Malzac@cesr.fr \\ 2 Laboratoire d'Astrophysique Observatoire de Grenoble, BP 53, 38041 Grenoble Cedex 9, France \\ 3 Service d'Astrophysique, CEA-Saclay, Bat. 709, L'Orme des Merisiers, 91191 Gif-sur-Yvette, Cedex, France \\ 4 APC-UMR 7164, 11 place M. Berthelot, 75231 Paris, France \\ 5 Cavendish Laboratory, University of Cambridge, Madingley Road, Cambridge CB3 OHE, UK \\ 6 AIM - Astrophysique Interactions Multi-échelles (Unité Mixte de Recherche 7158 CEA/CNRS/Université Paris 7 Denis Diderot), \\ CEA-Saclay, Bât. 709, L'Orme des Merisiers, 91191 Gif-sur-Yvette Cedex, France \\ 7 INAF - Osservatorio Astronomico di Brera, via E. Bianchi 46, 23807 Merate, Italy \\ 8 INTEGRAL Science Data Center, Chemin d'Écogia 16, 1290 Versoix, Switzerland
}

Received 10 June 2005 / Accepted 16 November 2005

\section{ABSTRACT}

We report the results of an observation of Cygnus X-1 performed on June 7-11, 2003 with INTEGRAL that we combine with simultaneous radio observations with the Ryle telescope. Both spectral and variability properties of the source indicate that Cygnus X-1 was in an Intermediate State. The INTEGRAL spectrum shows a high-energy cut-off or break around $100 \mathrm{keV}$. The shape of this cut-off differs from pure thermal Comptonisation, suggesting the presence of a non-thermal component at higher energies. The average broad band spectrum is well represented by hybrid thermal/non-thermal Comptonisation models. However, models with mono-energetic injection, or models with an additional soft component are favoured over standard power-law acceleration models. During the 4 day long observation the broad band (3-200 keV) luminosity varied by up to a factor of 2.6 and the source showed an important spectral variability. A principal component analysis demonstrates that most of this variability occurs through 2 independent modes. The first mode consists in changes in the overall luminosity on time scale of hours with almost constant spectra (responsible for $68 \%$ of the variance) that are strikingly uncorrelated with the variable radio flux. We interpret this variability mode as variations of the dissipation rate in the corona, possibly associated with magnetic flares. The second variability mode consists in a pivoting of the spectrum around $\sim 10 \mathrm{keV}(27 \%$ of the variance). It acts on a longer time-scale: initially soft, the spectrum hardens in the first part of the observation and then softens again. This pivoting pattern is strongly correlated with the radio (15 GHz) emission: radio fluxes are stronger when the INTEGRAL spectrum is harder We propose that the pivoting mode represents a "mini" state transition from a nearly High Soft State to a nearly Low Hard State, and back. This mini-transition would be caused by changes in the soft cooling photons flux in the hot Comptonising plasma associated with an increase of the temperature of the accretion disc. The jet power then appears to be anti-correlated with the disc luminosity and unrelated to the coronal power. This is in sharp contrast with previous results obtained for the Low Hard State, suggesting a different mode of coupling between the jet, the cold disc, and the corona in Intermediate States. From this interpretation we also infer that the bolometric luminosity jumps by a factor of about 2 during the transition hard to soft, suggesting a radiatively inefficient accretion flow in the Low Hard State.

Key words. gamma-rays: observations - black hole physics - radiation mechanisms: non-thermal - X-rays: binaries - radio continuum: stars - X-rays: individuals: Cygnus X-1

\section{Introduction}

Cygnus X-1 is the prototype of black hole candidates. Since its discovery in 1964 (Bowyer et al. 1965), it has been intensively observed by all the high-energy instruments, from soft X-rays to $\gamma$-rays. It is a persistent source most often observed in the so-called Low Hard State (hereafter LHS), characterised by a relatively low flux in the soft $\mathrm{X}$-rays $(\sim 1 \mathrm{keV})$ and a high flux in the hard X-rays $(\sim 100 \mathrm{keV})$. In the LHS, the high-energy spectrum can be roughly described by a power-law with spectral index $\Gamma$ varying in the range $1.4-2.2$, and a nearly exponential cut-off at a characteristic energy $E_{\mathrm{c}}$ of a few hundred keV (see e.g. Gierlinski et al. 1997). Occasionally, the source switches to the High Soft State (HSS). The high-energy power-law is then much softer $(\Gamma>2.4)$ and the bolometric luminosity is dominated by a thermal component peaking at a few keV. Finally, there are also Intermediate States (hereafter IMS) in which the source exhibits a relatively soft hard X-ray 
spectrum $(\Gamma \sim 2.1-2.3)$ and a moderately strong soft thermal component (Belloni et al. 1996; Mendez \& van der Klis 1997). The IMS often, but not always, appears when the source is about to switch from one state to the other. When it is not associated with a state transition, it is interpreted as a "failed state transition". Until 1998, the source used to spend nearly $90 \%$ of its time in the LHS. In the recent years however there have been more IMSs and soft states (see Zdziarski et al. 2002; Pottschmidt et al. 2003; Gleissner et al. 2004).

Simultaneous radio/X-ray and high-energy observations of Cygnus X-1 and other sources have shown that the X-ray LHS is correlated with a strong radio emission which is consistent with arising from a jet (Fender 2001). In contrast, during HSS episodes the source appears to be radio weak (Brocksopp et al. 1999). The presence of a compact jet in the LHS was confirmed by Stirling et al. (2001) who presented evidence for an extended and collimated radio structure on milliarsecond scales.

State transitions are generally interpreted as being associated with changes in the geometry of the accretion flow. In the HSS the geometrically thin optically thick disk (Shakura \& Sunyaev 1973) extends down to the last stable orbit. The spectrum is dominated by the thermal disc component and peaks at a few $\mathrm{keV}$. The hard X-ray emission is then believed to be produced in a non-thermal corona above and below the disc (Gierliński et al. 1999, hereafter G99). In the LHS the geometrically thin disc is truncated at a few hundred Schwarschild radii, the innermost part of the accretion flow forms a geometrically thick optically thin and hot disc (Shapiro et al. 1976); Narayan \& Yi 1994) where high-energy radiation is produced trough thermal Comptonisation. During spectral transitions to the HSS the inner radius of the cold accretion disk decreases. This reduction of the inner disc radius is associated with either the cold disk penetrating inside the hot inner flow, or the later collapsing into an optically thick accretion disk with small active regions of hot plasma on top of it (Zdziarski et al. 2002). In both cases the enhanced soft photon flux from the disk tends to cool down the hot phase, leading to softer spectra. An alternative possibility would be that in both LHS and HSS, the geometry is that of a corona above a cold standard accretion disc (Bisnovatyi-Kogan \& Blinnikov 1976; Haard \& Maraschi 1993). In the LHS the coronal plasma is essentially thermal and the cold disc faint because most of the power is transported away and dissipated in the corona and a strong outflow (Beloborodov 1999; Malzac et al. 2001; Merloni \& Fabian 2002; Ferreira et al. 2006), while in the HSS most of the power is dissipated into the cold disc and the corona is non-thermal.

In the LHS, the radio flux is then positively correlated with the soft X-ray emission (3-25 keV, Corbel et al. 2000, 2003; Gallo et al. 2003). The X-ray emitting region and the jet seems to be intrinsically associated. This led to the now widely accepted idea that the corona (or hot thick disc) of the LHS constitutes the base of the jet (Fender et al. 1999; Merloni \& Fabian 2002; Markoff et al. 2005).

Cygnus X-1 represents a prime target for the INTEGRAL mission (Winkler et al. 2003) launched in 2002 October 17, whose instruments offer an unprecedented spectral coverage at high-energy, ranging from $3 \mathrm{keV}$ to several MeV. Cygnus X-1 was extensively observed (1 Ms) during the calibration phase

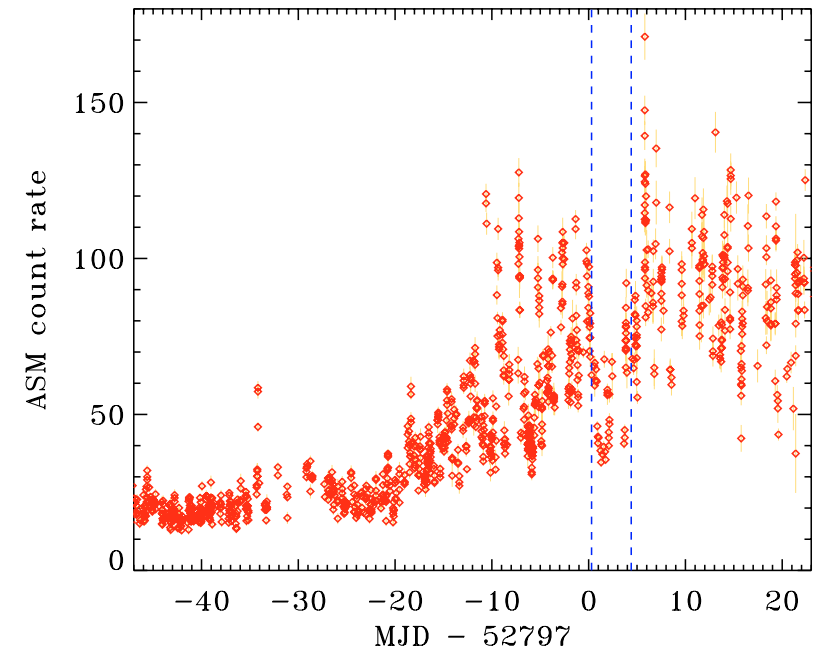

Fig. 1. RXTE/ASM light curve of Cygnus $\mathrm{X}-1$. The time of the INTEGRAL observation is delimited by the vertical dashed lines.

of the mission (Bouchet et al. 2003; Pottschmidt et al. 2003; Bazzano et al. 2003). At that time, the source presented all the characteristics of the LHS. The source was later observed again during the galactic plane survey and open-time programme, and occasionally found in IMSs (Cadolle Bel et al. 2006, hereafter CB05). In this paper we focus on the results of the first observation of Cygnus X-1 in the open time programme. This $300 \mathrm{ks}$ observation was performed on 2003 June 7-11 (rev 79/80) with a $5 \times 5$ dithering pattern ${ }^{1}$ (the effective exposure time was $275 \mathrm{ks}$ for $J E M-X 2,292 \mathrm{ks}$ for IBIS/ISGRI, and $296 \mathrm{ks}$ for $S P I)$. At this epoch, the RXTE All Sky Monitor count rate of Cygnus X-1 was higher than in typical LHS by up to a factor of 4 , and the light curve showed strong X-ray activity characteristic of state (or failed state) transitions (see Fig. 1). We also combine the INTEGRAL data with the results of coordinated radio observations $(15 \mathrm{GHz})$ performed with the Ryle telescope. In Sect. 2, we present a spectral analysis of the $J E M-X, I B I S / I S G R I$ and SPI spectra averaged over the whole duration of the observation, in Sect. 3 we study the strong broad band variability of the source during the observation.

\section{Average spectrum}

\subsection{Data processing}

We reduced the IBIS/ISGRI and JEM- $X$ data with the standard analysis procedure of the Off-Line Scientific Analysis OSA 4.2. released by the ISDC, whose algorithms are described in Goldwurm et al. (2003) and Westergaard et al. (2003) for $I B I S$ and $J E M-X$ respectively. A basic selection was performed to exclude those pointings too close to radiation belt entry or exit or spoilt by too large noise. The SPI data were preprocessed with OSA 4.2 using the standard energy calibration gain coefficients per orbit and excluding bad quality pointings which have anomalous exposure and dead time values,

\footnotetext{
INTEGRAL observations are made of a succession of exposures of about 30 min duration with varied pointed directions to enable SPI image deconvolution. Such a $30 \mathrm{~min}$ pointing is called a science window.
} 

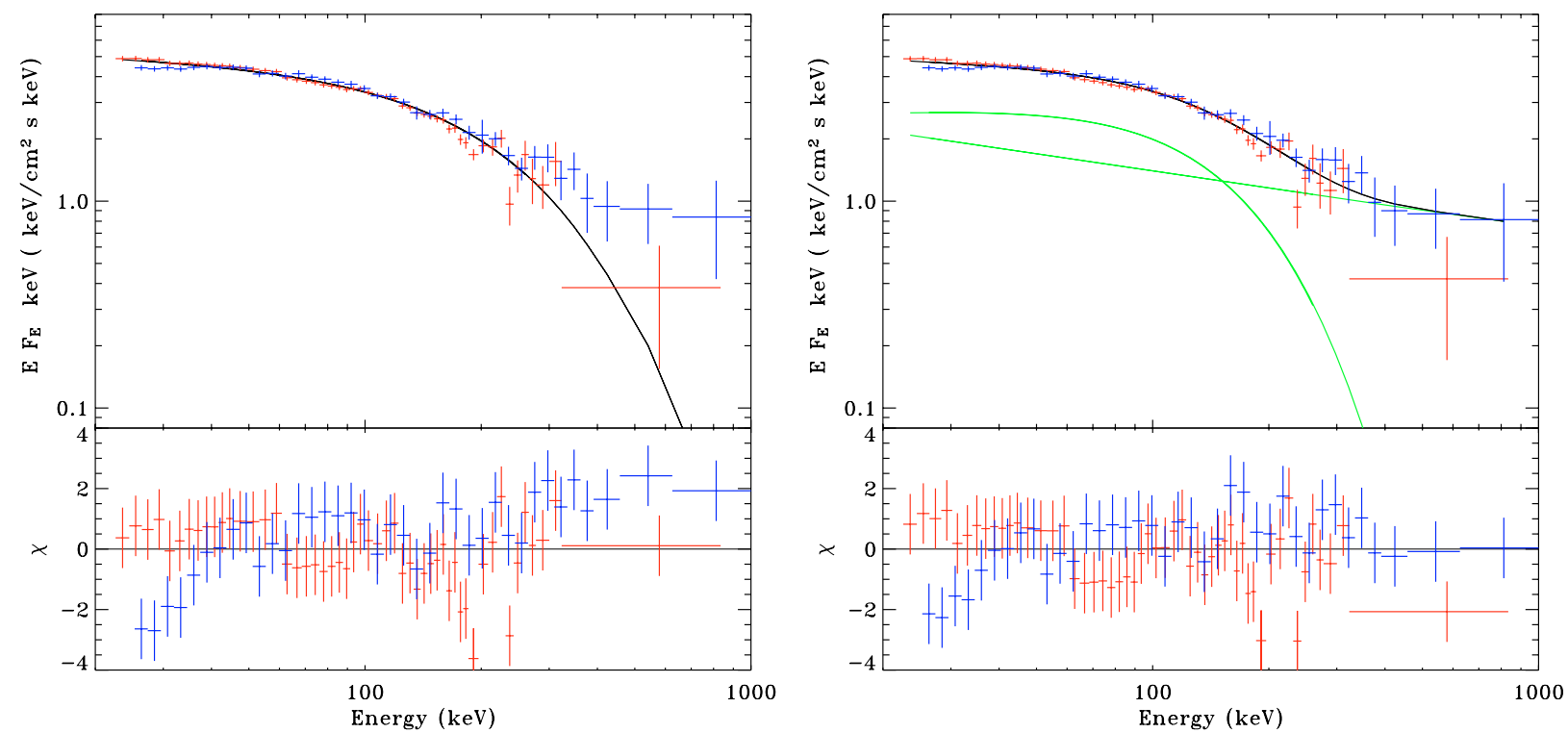

Fig. 2. Best fits of the IBIS/ISGRI (red) and SPI (blue) data with a pure comptonization model (CoMPPS, left) and Comptonisation plus powerlaw (right).

or with a high final chi-squared during imaging. SPIROs 9.2 (Skinner \& Connell 2003) was used to extract the spectra of Cygnus X-1, Cygnus X-3 and EXO 20390+375, with background model proportional to the saturating event count rates in the Ge detector. Concerning the instrumental response, version 15 of the IRF (Image Response Files) and version 2 of the RMF (Redistribution Matrix Files) were used.

We produced JEM-X (3-25 keV), ISGRI (20-800 keV) and SPI (25-1000 keV) spectra averaged over revolution 79 and 80. Uncertainties of 3\% were added in quadrature to all three spectra to account for systematic errors. The resulting spectra were fitted using XSPEC V11.3.1.

\subsection{JEM-X spectrum}

The best power-law fit to the 3-20 keV JEM-X spectrum has a spectral index $\Gamma=2.16$ which is intermediate between LHS and HSS spectral indices. The fit is however not statistically acceptable $\left(\chi^{2} / v=611 / 131\right)$ with strong residuals indicating the presence of strong reflection and broad iron line features and a soft component (see below in Sect. 2.4 and Fig. 3).

\subsection{SPI and IBIS/ISGRI spectra}

The spectra show a highly significant evidence for a cut-off (or at least a break) around $100 \mathrm{keV}$ (see Fig. 2). We first fit the $I S G R I$ and SPI spectra independently with the Comptonisation model coMPPS (Poutanen \& Svensson 1996) The coMPPS model provides a reasonable fit with reduced $\chi^{2}$ values 0.82 and 1.12 for $I S G R I$ and SPI respectively. The best-fit parameters obtained for both instruments (shown in Table 1) are compatible within the error bars.

However the residuals show an excess emission with respect to the Comptonisation model above the thermal cutoff in the SPI spectrum, which is not detected in the ISGRI

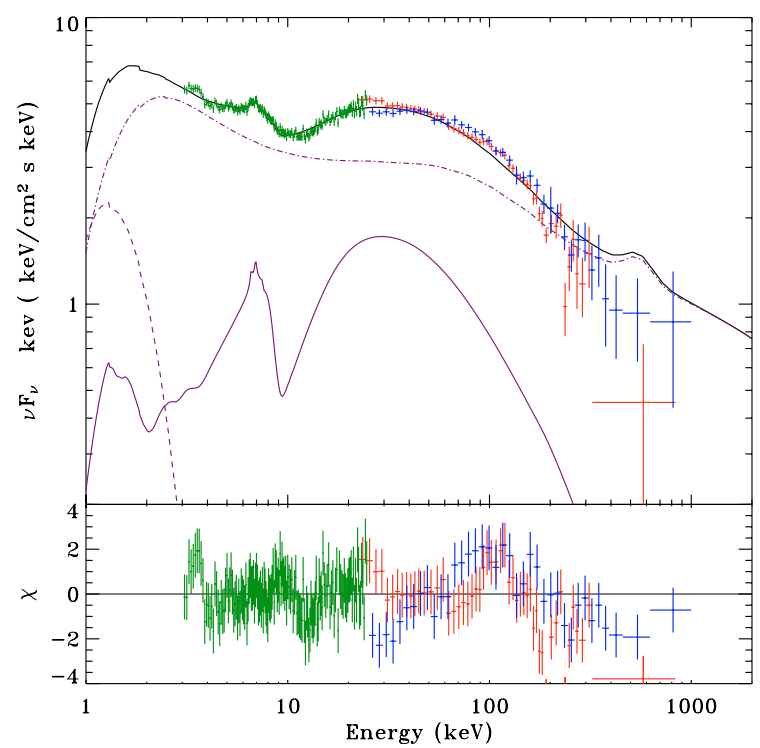

Fig. 3. Joint $J E M-X / S P I / I S G R I$ spectrum of Cygnus $\mathrm{X}-1$ averaged over revolutions 79 and 80 . The data are fitted with the thermal/non-thermal hybrid Comptonisation model EQPAIR with power-law injection of relativistic electrons (see text and Table 2). The lighter curves show the reflection component (solid), the disc thermal emission (dashed) and the Comptonised emission (dot-dash). The green, red and blue crosses show the $J E M-X$, IBIS/ISGRI and SPI data respectively.

spectrum. This prompted us to reprocess the SPI data using different background models based on an empty field observation to model the uniformity map of the detection plan. We also tested using the instrument team processing software instead of OSA. This did not affect the presence of a high energy excess in the $S P I$ spectrum. We concluded that the non detection of this excess by $I S G R I$ was attributable to its poorer sensitivity above $200 \mathrm{keV}$. This is also the reason why the ISGRI fit appears statistically better. Within the errors bars the highest energy IBIS and SPI data points are not too far and still compatible. 
Table 1. Best-fit parameters of the SPI and IBIS spectra fitted with thermal-Comptonisation and thermal-Comptonisation plus power-law models. For each instrument the first line gives the results of the COMPPS model alone (Comptonisation tamperature $k T$ and Thomson depth $\tau$ ) while the second line gives the results of the fit with compPs plus a power-law (photon index $\Gamma$ ). In the compPs model the black body temperature of the soft seed photons was fixed at $0.1 \mathrm{keV}$ in all fits.

\begin{tabular}{ccccc}
\hline \hline data & $k T(\mathrm{keV})$ & $\tau$ & $\Gamma$ & $\chi^{2} / v$ \\
\hline ISGRI & $79_{-7.3}^{+7.5}$ & $1.06_{-0.14}^{+0.17}$ & & $46 / 56$ \\
ISGRI & $51_{-12}^{+12}$ & $2.01_{-0.81}^{+0.59}$ & $2.39_{-1.57}^{+0.14}$ & $43 / 54$ \\
SPI & $90_{-8}^{+18}$ & $0.98_{-0.24}^{+0.15}$ & & $50 / 44$ \\
SPI & $45_{-12}^{+15}$ & $2.38_{-0.49}^{+1.1}$ & $2.06_{-0.70}^{+0.24}$ & $23 / 42$ \\
SPI+ISGRI & $84_{-6.8}^{+6.3}$ & $1.00_{-0.10}^{+0.14}$ & & $142 / 102$ \\
SPI+ISGRI & $42_{-4}^{+15}$ & $2.64_{-0.33}^{+0.50}$ & $2.27_{-0.77}^{+0.66}$ & $106 / 100$ \\
\hline
\end{tabular}

To obtain better constraints on the parameters we then fit simultaneously the SPI and ISGRI data. The results are shown in Fig. 2. In all fits we allow for a free normalisation constant of the model for each instrument to correct for inter-calibration errors. The resulting difference in normalisation between the instruments never exceeds $20 \%$. The simple coMPPS model leads to best-fit parameters that are intermediate between those obtained for the individual ISGRI and SPI spectra, but the statistical quality of the fit is not as good $\left(\chi^{2} / v=1.4\right)$. As a further test for the presence of a high-energy excess, we refit both spectra with COMPPS adding a power law component. When we allowed for this additional component the reduced $\chi^{2}$ decreased to 1.05. This improvement is highly significant, a F-test $\left(\Delta \chi^{2}=36\right.$ for 2 additional parameters) shows that the probability that this improvement occurred by chance is $5 \times 10^{-7}$. We conclude that either the high-energy cut-off is not due to thermal Comptonisation (e.g. associated to a cut-off in a non-thermal lepton distribution) or there is an additional nonthermal component at high-energy. We stress that the presence of the high energy excess is not in conflict with the ISGRI data although this instrument was not able to detect it.

\subsection{Joint spectrum}

We now consider the joint JEM-X/ISGRI/SPI spectrum. The simple models considered in the previous section do not provide an acceptable description of the broad band spectrum: strong reflection features and a soft-excess are required by the JEM-X data. CB05 present spectral fits of the same data with various thermal and non-thermal Comptonisation models. In these models the soft excess in the JEM-X spectrum is accounted for by thermal emission of the accretion disc. However, although, these fits are statistically acceptable, they require high temperatures of the accretion disc (1.21 \pm $0.29 \mathrm{keV})$. As mentioned in CB05 such a high temperature of the accretion disc is not realistic. Since the distance and scale of the accretion disc (i.e. mass of the black hole) are quite well known for this source, the intrinsic luminosity of the accretion disc is constrained: if the accretion disc in Cygnus X-1 had an inner temperature of $1 \mathrm{keV}$, it would produce a flux at least one order of magnitude larger than what is observed.

In order to get a more physically motivated representation of the data, we investigate in more details thermal/nonthermal hybrid Comptonisation models and attempt to fit the data using different variants of the EQPAIR model (Coppi 1999; G99; Frontera et al. 2001 (hereafter F01); Zdziarski et al. 2002, 2004) where the accretion disc temperature is arbitrarily fixed to a reasonable value. This model was shown to be successful in accounting for the high energy spectra of Cygnus X-1 and other black holes candidates in different spectral states and over a very broad energy band ranging from soft X-rays to gammarays (see e.g. McConnell et al. 2000, 2002). The wide use of this model in the literature will allow us to compare our observation with previously published results.

\subsubsection{The hybrid thermal/non-thermal comptonisation model (EQPAIR)}

A detailed description of the EQPAIR model can be found in G99. Its main ingredient is a spherical hot plasma cloud with continuous acceleration of electrons intended to model the emission of the hot disc/coronna. The high-energy electrons lose energy because of Compton, Coulomb, and bremsstrahlung processes and thus establish a steady-state distribution. At high energies, the distribution is non-thermal (power-law like), but at low energies a Maxwellian distribution is established. The temperature of the Maxwellian population, $k T_{\mathrm{e}}$ is determined by balance between Compton gains and losses, Coulomb heating by high energy electrons, bremsstrahlung losses, and direct heating (e.g., Coulomb heating by energetic ions). The total number of electrons (not including $\mathrm{e}^{+}-\mathrm{e}^{-}$pairs, the production of which is also taken into account) is determined by the corresponding Thomson optical depth $\tau_{\mathrm{p}}$ which is a free parameter. The cloud is illuminated by soft thermal photons emitted by an accretion disk. These photons serve as seed for Compton scattering by both thermal and nonthermal electrons. The system is characterised by the power $L_{i}$ supplied to its different components. We express each of them dimensionlessly as a compactness, $l_{i}=L_{i} \sigma_{\mathrm{T}} /\left(\mathcal{R} m_{e} c^{3}\right)$. $\mathcal{R}$ is the characteristic dimension of the plasma, $\sigma_{\mathrm{T}}$ is the Thomson cross-section. $l_{\mathrm{s}}, l_{\mathrm{th}}, l_{\mathrm{nth}}$, and $l_{\mathrm{h}}=l_{\mathrm{th}}+l_{\text {nth }}$ correspond to the power in soft disk photons entering the plasma, thermal electron heating, electron acceleration and the total power supplied to the plasma. We follow G99 and set $l_{\mathrm{s}}=10$.

The disc spectrum incident on the plasma is modelled as coming from a pseudo-Newtonian accretion disk extending from $R_{\text {out }}=10^{3} R_{\mathrm{g}}$ down to the minimum stable orbit, $R_{\text {in }}=6 R_{\mathrm{g}}$. Its spectral shape is then characterised by the maximum colour temperature of the disk, $k T_{\max }$. Previous observations of Cygnus $\mathrm{X}-1$ with $\mathrm{X}$-rays telescopes indicate temperatures ranging from $0.1 \mathrm{keV}$ in the LHS to up to $0.6 \mathrm{keV}$ in the HSS (G99; F01). For such temperatures the peak of the thermal disk emission is below the energy range covered by INTEGRAL, and the disk temperature cannot be constrained by our observations, we therefore fixed $k T_{\max }=0.3 \mathrm{keV}$. The covering factor of the corona is unity. 
The spectrum from both reflection and the $\mathrm{Fe} \mathrm{K}_{\alpha}$ is calculated, taking into account relativistic smearing with the emissivity dependence $\propto R^{-2}$. The reflecting material is allowed to be ionised, with the degree of ionisation characterised by the ionisation parameter $\xi$. We follow G99 and fix the column density of absorbing material along the line of sight to $N_{\mathrm{H}}=5 \times 10^{21} \mathrm{~cm}^{-2}$ and the inclination angle of the system at 45 degrees.

\subsubsection{Fit results}

We first performed a fit similar to those of G99 and F01 with the non-thermal electrons injected with a power-law distribution of Lorentz factors ranging from $\gamma_{\min }=1.3$ to $\gamma_{\max }=1000$. The upper and lower limits $\gamma_{\min }$ and $\gamma_{\max }$ where kept fixed while fitting for the power-law index $\Gamma_{\mathrm{p}}$. This results in an acceptable fit with a reduced $\chi^{2}$ of 1.29 . The unfolded broadband spectrum and residuals are shown in Fig. 3. The best-fit parameters are presented in Table $2 . l_{\mathrm{h}} / l_{\mathrm{s}}$ is about unity i.e. intermediate between what is generally found in the LHS (4-10, see Ibragimov et al. 2005) and the HSS $(\lesssim 0.4)$, the heating of the plasma is dominated by the non-thermal acceleration $\left(l_{\text {nth }} / l_{\mathrm{h}}=1\right)$. Overall the parameters are similar to those obtained by G99 for the IMS of 1996 May 23. However an inspection of the residuals (Fig. 3) shows that the high energy part of the ISGRI/SPI spectrum is not well represented by this model. The model does not provide a good description of the shape of the high-energy cut-off and overestimates the measured flux above $200 \mathrm{keV}$. As an alternative we attempted to fit the spectrum assuming that the electrons are injected at a single Lorentz factor $\gamma_{\text {inj }}$ instead of a power-law distribution. Such a monoenergetic injection is not expected in the case of shock acceleration but could be achieved in reconnection events that are expected to power the corona. The resulting fit is displayed in Fig. 4 and the unabsorbed best fit model spectrum is shown in Fig. 13. The best-fit parameters are shown in Table 2. Note that the fraction of non-thermal power is now only $l_{\text {nth }} / l_{\mathrm{h}}=0.51$. This model gives a good description of the INTEGRAL spectrum. The best-fit value for the maximum Lorentz factor of the electrons $\gamma_{\mathrm{inj}}=8.6$ implies that the once scattered soft photons reach a maximum energy of about $4 \gamma_{\mathrm{inj}}^{2} k T_{\max } \sim 90 \mathrm{keV}$. In other words, in this model the high-energy cut-off observed in the spectrum is non-thermal and corresponds to the upper end of the non-thermal electron distribution.

So far we have shown that the soft excess in the JEM-X data does not require an unphysical high accretion disc temperature and can be accounted for by an hybrid particle distribution. In this context, our data favour mono-energetic injection over power-law acceleration. However, this is not the only possible interpretation. Indeed, such a soft excess is not uncommon in IMSs and the softest LHSs. It is often interpreted as a hot spot on the accretion disc or alternatively as a component due Comptonisation in the warm upper layers of the disc. It can be accounted for by adding a second Comptonisation component with a temperature of a few keV (Di Salvo et al. 2001; F01; Zicky et al. 2001). Alternatively, Markoff et al. (2005),
Table 2. Best-fit parameters of the joint JEM-X, SPI and IBIS/ISGRI spectra with hybrid thermal/non-thermal Comptonisation models (EQPAIR, see Sect. 2.4). The temperature of the inner disk (DISKPN) was fixed to $k T_{\max }=0.3 \mathrm{keV}$ in all fits. The soft photon compactness is fixed at $l_{\mathrm{s}}=10$. The absorbing column density is $N_{\mathrm{h}}=5 \times 10^{21}$ and the inclination angle 45 degrees. The fit parameter $\tau_{\mathrm{p}}$ refers to the Thomson optical depth of the electrons associated with ions. The table also gives the resulting total optical depth $\tau_{\mathrm{T}}$, including electronpositron pairs whose production is calculated self-consistently, and the temperature $k T_{\mathrm{e}}$ of the thermalised particles, computed according to energy balance. The extrapolated $0.1-1000 \mathrm{keV}$ model flux, $F_{\text {bol }}$, and thermal disc component flux, $F_{\text {disc }}$, are given in units of $10^{-8} \mathrm{erg} \mathrm{s}^{-1} \mathrm{~cm}^{-2}$. The first column shows the results of the fit with a power law injection of non-thermal electrons with Lorentz factors ranging from $\gamma_{\min }=1.3$ to $\gamma_{\max }=1000$. The data are then fitted for the index of the distribution of injected electrons $\Gamma_{p}$. The second column shows the best fit parameters for a mono-energetic injection of electrons, the fit parameter $\gamma_{\mathrm{inj}}$ represents the Lorentz factor of the injected particles. Finally, the third column gives the results for a power-law injection plus an additional warm Comptonisation component (COMPTT). Its best fit temperature $k T_{\text {comptt }}$, Thomson depth $\tau_{\text {comptt }}$ and flux $F_{\text {comptt }}$ are also shown. In this fit $\Gamma_{p}$ pegged to its minimum allowed boundary (2).

\begin{tabular}{cccc}
\hline \hline Model & EQPAIR pow. & EQPAIR mono. & EQPAIR+COMPTT \\
\hline$l_{\mathrm{h}} / l_{\mathrm{s}}$ & $1.19_{-0.06}^{+0.07}$ & $0.85_{-0.03}^{+0.02}$ & $3.06_{-0.16}^{+0.41}$ \\
$l_{\text {nth }} / l_{\mathrm{h}}$ & $1_{-0.04}^{+0}$ & $0.51_{-0.04}^{+0.04}$ & $0.23_{-0.02}^{+0.37}$ \\
$\tau_{\mathrm{p}}$ & $1.31_{-0.04}^{+0.02}$ & $0.55_{-0.06}^{+0.01}$ & $0.79_{-0.07}^{+0.13}$ \\
$\Gamma_{\mathrm{p}}$ or $\gamma_{\text {inj }}$ & $2.68_{-0.01}^{+0.02}$ & $8.41_{-0.92}^{+0.62}$ & $2_{-0}^{+0.21}$ \\
$\Omega / 2 \pi$ & $0.67_{-0.02}^{+0.09}$ & $0.71_{-0.03}^{+0.09}$ & $0.62_{-0.06}^{+0.09}$ \\
$\xi\left(\mathrm{erg} \mathrm{cm} \mathrm{s}^{-1}\right)$ & $2063_{-367}^{+463}$ & $525_{-84}^{+143}$ & $991_{-176}^{+297}$ \\
$E_{\text {line }}(\mathrm{keV})$ & $6.76_{-0.33}^{+0.36}$ & $7.02_{-0.23}^{+0.32}$ & $6.67_{-0.25}^{+0.25}$ \\
$\mathrm{EW}(\mathrm{eV})$ & $55_{-34}^{+33}$ & $90_{-24}^{+38}$ & $76.3_{-29}^{+34}$ \\
$k T_{\text {comptt }}(\mathrm{keV})$ & & & $2.53_{-0.99}^{+0.20}$ \\
$\tau_{\text {comptt }}$ & & & $2.73_{-0.27}^{+0.34}$ \\
$F_{\text {comptt }}$ & & & 1.52 \\
$F_{\text {disc }}$ & 1.9 & 3.0 & 0.63 \\
$F_{\text {bol }}$ & 7.5 & 7.9 & 5.9 \\
$k T_{\mathrm{e}}(\mathrm{keV})$ & 20.3 & 50 & 65 \\
$\tau_{\mathrm{T}}$ & 1.36 & 0.55 & 1.01 \\
$\chi^{2} /$ d.o.f. & $316 / 245$ & $244 / 245$ & $216 / 242$ \\
\hline
\end{tabular}

suggest that this soft component originates from optically thin synchrotron emission in the jet.

To check the effect of such an additional soft component on the derived parameters of the EQPAIR model, we fit the spectrum with a model consisting in the power-law injection EQPAIR model, including the same $0.3 \mathrm{keV}$ thermal component, relativistically smeared iron line and ionised reflection component as in our previous models (see Sect. 2.4.1) plus an additional Comptonisation component (COMPTT model in XSPEC) in a warm medium.

This led to an excellent fit of the INTEGRAL data, with $\chi^{2} / v=0.89$, that is shown in Fig. 5 and Table 2. The additional Comptonisation component has a best-fit temperature of $2.5 \mathrm{keV}$ and a Thomson optical depth of 2.7. The fit is much 


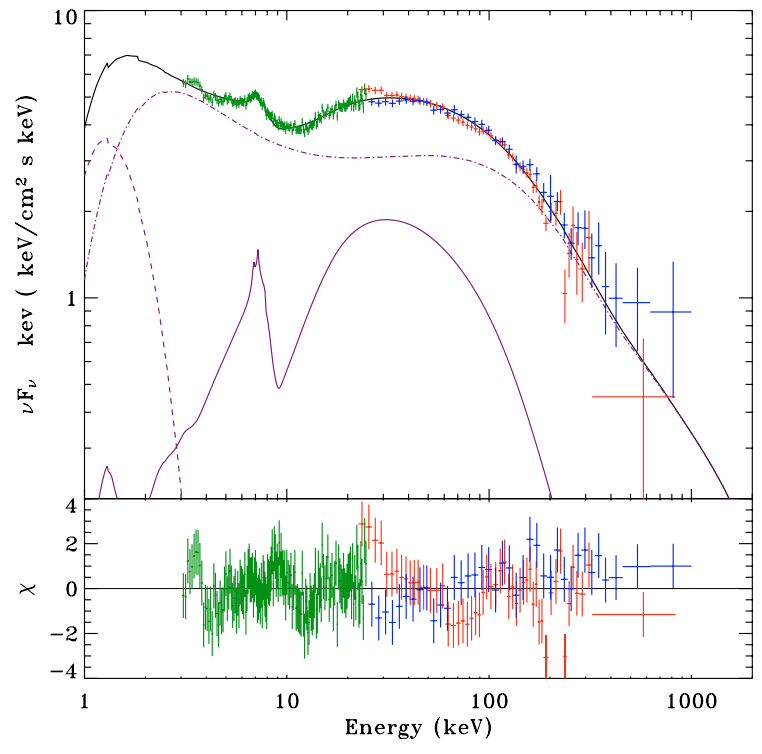

Fig. 4. Same as in Fig. 3 except that the fitting model is now EQPAIR with mono-energetic injection of relativistic electrons with Lorentz factor $\gamma_{\mathrm{inj}}=8.6$ (see text and Table 2).

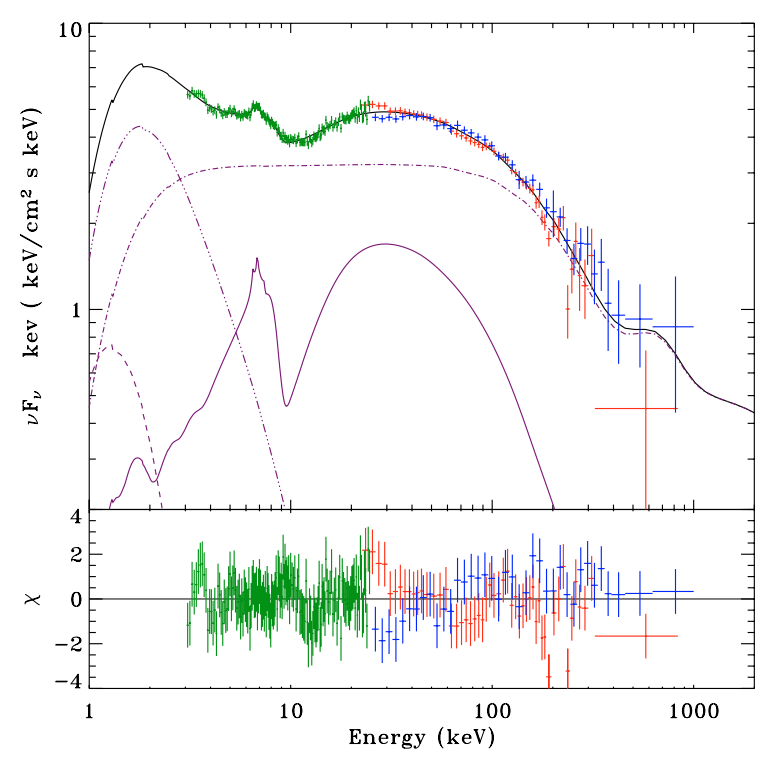

Fig. 5. Same as in Fig. 3 except that the fitting model is now EQPAIR with a power-law injection relativistic electrons plus an additional warm Comptonisation soft component (COMPTT, see text and Table 2). The COMPTT component is shown by the 3-dot-dashed curve.

better than what obtained with the powerlaw injection EQPAIR model. The probability that the improvement obtained when the soft COMPTT component is added to the powerlaw injection EQPAIR model occured by chance is $7 \times 10^{-20}$ according to F-test $\left(\Delta \chi^{2}=100\right.$ for 3 additional parameters). This fit is statistically comparable to that obtained with mono-energetic injection. It demonstrates that mono-energetic injection is far from inevitable.

We also note an interesting difference between power-law and mono-energetic injection models: the presence of a broad $\mathrm{e}^{+}-\mathrm{e}^{-}$pair annihilation line around $511 \mathrm{keV}$ that is clearly visible in Figs. 3 and 5 (power-law injection) but absent from Fig. 4 (mono-energetic injection). In the power-law model some electrons are injected at very high Lorentz factors $(\lesssim 1000)$ these electrons efficiently up-scatter soft-photons at energies above the pair production threshold, leading to a pair cascade and subsequent annihilation, whereas in the monoenergetic case the pair content of the plasma is negligible due to the much lower energy of the electrons $(\leqslant 8.41)$. Unfortunately the poor statistics of the data does not allow us to discriminate between these models on the basis of the annihilation line.

In the EQPAIR+COMPTT spectral model the fraction of nonthermal power $\left(l_{\text {nth }} / l_{\mathrm{h}}\right)$ is reduced to about $20 \%$ (to be compared to $100 \%$ and $50 \%$ obtained respectively for the simple power-law and mono-energetic injection models). This shows that the measured non-thermal fraction is sensitive to the assumptions of the spectral model. In the latter fit the hot plasma is essentially thermal, in agreement with the conclusions of CB05. Indeed, the equilibrium temperature and optical depth we obtain are similar to what obtained by these authors fitting the same data with a simple thermal Comptonisation model plus a multicolour disc spectrum. We also note that in the EQPAIR + COMPTT fit, the hard to soft compactness ratio is larger $\left(l_{\mathrm{h}} / l_{\mathrm{s}} \simeq 3\right.$ instead of $\simeq 1$ in the previous fits). Overall the parameters are closer to what is usually obtained in the LHS.

In short, our spectral analysis does not favour the simple power-law injection EQPAIR model. Rather, models including an additional soft component (and a weak non-thermal fraction), or alternatively, models with an important thermal fraction but mono-energetic injection are preferred. There are however some caveats in our spectral analysis that might affect any conclusion drawn from our fits: First, there are still open calibration issues, in particular regarding the $\mathrm{X}$-ray monitor JEM-X specially below $5 \mathrm{keV}$. It is in this energy range that the soft component shows up and it is very important to constrain the parameters of the hybrid model. Second, our model assumes a unique emitting zone although it is likely that in an IMS the situation is more complex. Indeed, the thermal hot flow of the LHS probably coexists with the non-thermal corona of the HSS in distinct regions of the accretion flow. If various emitting regions, with very different spectra and physical parameters, contribute to the observed spectrum, then the derived best-fit parameters have a poor physical significance. Similarly, and perhaps more importantly, the parameters determining the shape of the spectrum vary not only within the accretion flow but also with time. As we will show below, the source exhibited a strong spectral variability during the observation with the appearance of spectra ranging from quasi-LHS to quasi-HSS. Under such circumstances, the precise value of the best-fit parameters of the average spectrum may be physically irrelevant.

\section{Spectral variability}

\subsection{Set up}

In order to study the spectral variability of the source during the observation, we produced light curves in 16 energy bands ranging from 3 to $200 \mathrm{keV}^{2}$ with a time resolution of the duration

2 Namely: 3-4, 4-5, 5-6, 6-7, 7-9, 9-11, 11-13, 13-15, 15-20, 20-30, 30-40, 40-50, 50-80, 80-100, 100-140 and 140-200 keV. 
of a science window (i.e. $30 \mathrm{~min}$ ). Above $200 \mathrm{keV}$, the variability is dominated by statistical noise. The count rate in each band was then renormalised so that its time average matches the energy flux calculated from the best-fit model of the joint average JEM-X/ISGRI/SPI spectrum shown in Fig. 5. Namely, for each energy band we compute the quantity

$F(t)=\frac{C(t)}{\bar{C}} \bar{F}$,

where $C(t)$ is the mean count rate during pointing $t, \bar{C}$ is the count rate averaged over the whole observation, $\bar{F}$ is the observation average energy flux in this band given by the best fit model. We use $F(t)$ as a proxy for the instantaneous energy flux, therefore neglecting the effects of the spectral variations on the instrumental response. This simple deconvolution method provides us with a convenient approximate of the broadband energy spectrum for each pointing that will be useful for a physical interpretation of the variability. This method is much more convenient than fitting the spectra for each science window, in particular if one considers the large number of parameters required to fit the data and the poor statistic in the short exposure spectra. On the other hand our method for estimating the spectra from the light curves also enables us to improve the photon statistics by combining the IBIS/ISGRI and $S P I$ instruments in the energy range where they overlap. The time dependent flux $F(t)$ in an overlapping band is estimated as follows:

$F(t)=\frac{\sigma_{\mathrm{S}}^{2} F_{\mathrm{I}}(t)+\sigma_{\mathrm{I}}^{2} F_{\mathrm{S}}(t)}{\sigma_{\mathrm{S}}^{2}+\sigma_{\mathrm{I}}^{2}}$.

Where $F_{\mathrm{I}}$ and $F_{\mathrm{S}}$ are the measured ISGRI and SPI fluxes in that band; $\sigma_{\mathrm{I}}$ and $\sigma_{\mathrm{S}}$ are their time averaged uncertainties. This combination minimises the average uncertainty on $F$.

The resulting light curves are shown in Fig. 6. The time averaged $3-200 \mathrm{keV}$ model flux is $\bar{F}_{3-200}=2.87 \times$ $10^{-8} \mathrm{erg} \mathrm{cm}^{-2} \mathrm{~s}^{-1}$. The energy flux has a rms amplitude of $16 \%$, and the ratio of the maximum to the minimum luminosity is 2.6.

\subsection{Principal Component Analysis (PCA)}

The light curves shown in Fig. 6 exhibit a complex and strong broad band variability of the spectra as well as the overall flux. We use a principal component analysis (PCA) to seek for variability patterns in our sample. PCA is a powerful tool for multivariate data analysis used for a broad range of applications in natural as well as social sciences (see e.g. Kendall 1980). It has also been used in astronomy. For instance, Francis \& Wills (1999) provide a brief introduction to PCA as applied to quasar spectra. Previous application of PCA to spectral variability include Mittaz et al. (1990) who discussed the UV variability of NGC 4151 and Vaughan \& Fabian (2004) for the X-ray variability of MCG6-30-15.

The main use of PCA is to reduce the dimensionality of a data set while retaining as much information as possible. It transforms a number of (possibly) correlated variables into a (smaller) number of uncorrelated variables called principal components. These principal components may define patterns

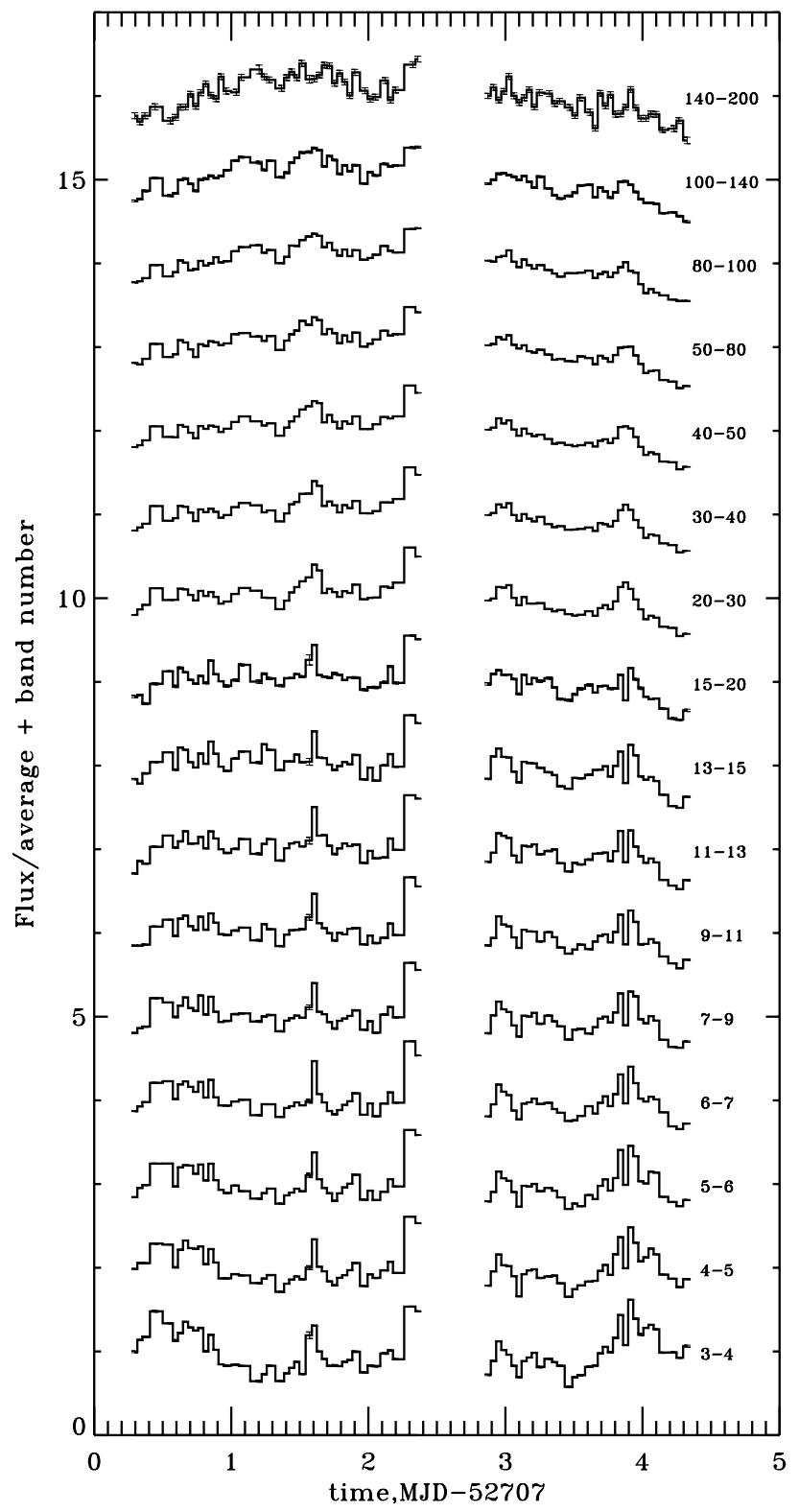

Fig. 6. Light curves in different $J E M-X$ an $I S G R I / S P I$ bands with increasing photon energy from bottom to top, as labelled in keV. Each light curve was re-scaled to the average flux and then incremented according to its energy range for clarity.

or correlations in the data set that can often be interpreted more easily than the original (large) data sets.

In our specific application we have $p=79$ spectra measured at times $t_{1}, t_{2}, \ldots, t_{p}$ and bined into $n=16$ bins corresponding to energies $E_{1}, E_{2}, \ldots, E_{n}$. Each spectrum can be thought of as a point in a $n$-dimensional space, so that the coordinate of the point $j$ along the $E_{i}$ axis is given by the corresponding energy flux $F\left(t_{j}, E_{i}\right)$. The shape and extension of the cluster of points formed by the whole set of spectra characterises the source variability. The idea behind PCA is to determine a new coordinate system in which the description of this cluster will be simpler.

For this purpose, the data can be viewed as the $p \times n$ matrix which coefficients are given by the energy fluxes $F\left(t_{j}, E_{i}\right)$. Then 
one can compute the $n \times n$ covariance matrix of the data. In practice, PCA consists in diagonalizing this covariance matrix to obtain its eigenvalues and eigenvectors. This procedure gives the coordinates of each eigen vector i.e. the $C_{k}\left(E_{i}\right)$ coefficients denoting the coordinate of the $k$ th eigenvector along $E_{i}$ axis.

These eigenvectors define a new coordinate system, in this $n$-dimensional parameter space, which best describes the variance in the data. The first principal component, or PC 1 (the eigenvector with the highest eigenvalue), marks the direction through the parameter space with the largest variance. The next Principal Component (PC 2) marks the direction with the second largest amount of variance, etc.

Let $\alpha_{k}\left(t_{j}\right)$ be the new coordinate of the spectrum $j$ along the $k$ th eigen vector, then the relation between the two coordinate systems can be written as follows:

$F\left(t_{j}, E_{i}\right)=\bar{F}\left(E_{i}\right)+\sum_{k=1}^{n} \alpha_{k}\left(t_{j}\right) C_{k}\left(E_{i}\right)$,

where $\bar{F}\left(E_{i}\right)$ is the time averaged flux at energy $E_{i}$ (the time averaged spectrum is used as the origin for the eigenvector coordinate system).

Equation (3) amounts to a linear decomposition into $n$ independent components of the variability (the eigenvectors $C_{1}$, $\left.C_{2}, \ldots, C_{n}\right)$. The normalisation coefficients of each PCA component (respectively $\alpha_{1}, \alpha_{2}, \ldots, \alpha_{n}$ ) vary in time. Their fluctuations account for the sample variance. On the other hand the eigenvectors $C_{k}$ are constant, they define the variability mode of each PCA component. Since the eigenvectors describe fluctuations around the time-average flux, both the $\alpha_{k}$ and $C_{k}$ coefficients can take negative as well as positive values. If the $C_{k}$ coefficients are positive at all energies, the variability mode associated to component $k$ can be understood as due to an additive spectral component with a fixed shape and a variable normalisation. When the sign of $C_{k}$ depends on energy, this corresponds to more complex spectral variability modes (e.g. pivoting).

As mentioned above, the PCA components are ordered according to the amount of sample variance they account for (i.e. the observed fluctuations of $\alpha_{1}$ cause more variance than those of $\alpha_{2}$ which produce more variance than $\alpha_{3}$ etc.). The first few Principal Components (those representing most of the variance in the data) should reveal the shape of the relevant spectral components or variability modes. The weaker Principal Components might be expected to be dominated by the statistical and systematic noise in the spectra. To summarise, PCA finds the decomposition that maximises the variability due to lower order components, so that most of the variability can be described using a small number of components.

\subsection{Results of the PCA analysis}

The results of our PCA analysis of the spectral variability of Cygnus X-1 are illustrated in Fig. 7, which shows how the 2 first principal components affect the flux and spectrum and their respective contribution to the total observed variance as a function of energy. As can be seen in this figure, the first principal component (PC 1) consists in a variability mode

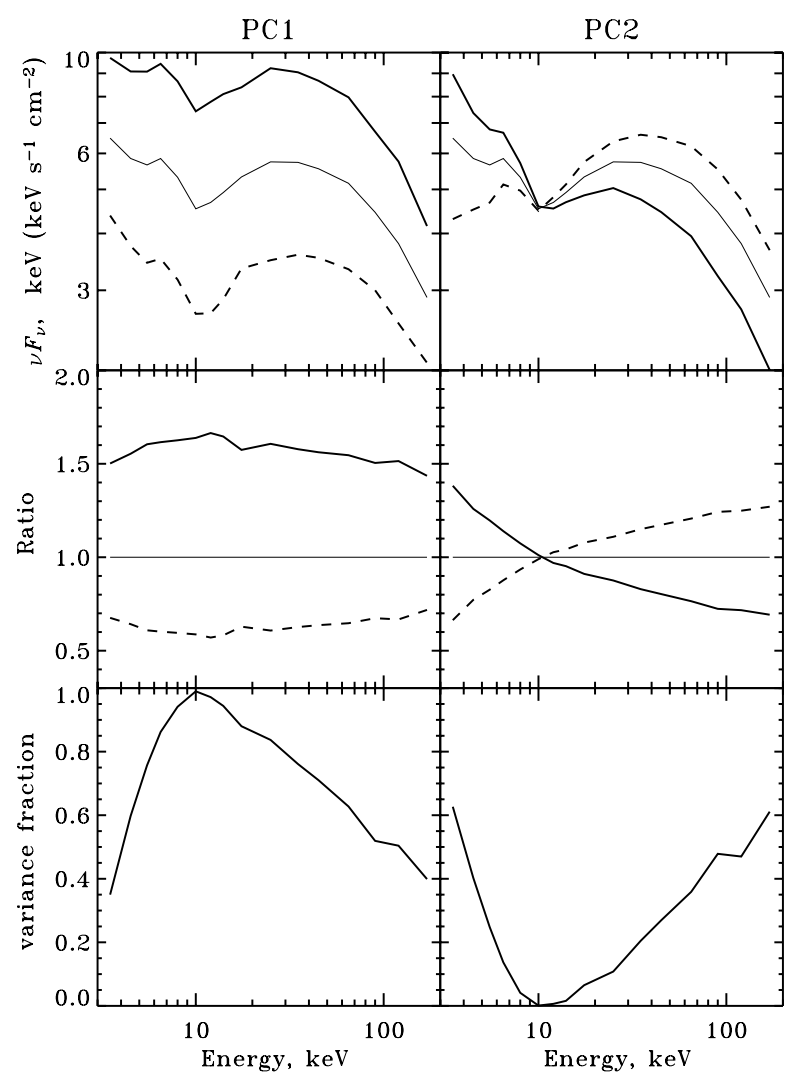

Fig. 7. The 2 first principal components of variability. The upper panels illustrate the effects of the each component on the shape and normalisation of the spectrum: time average spectrum (light line) and spectra obtained for the maximum (thick solid line) and minimum (dashed line) observed values of the normalisation parameter. The middle panels show the ratio of spectra obtained of the maximum and minimum spectra to the average one. The bottom panels show the contribution of each component to the total variance as a function of energy.

dominated by variations in the luminosity (normalisation) with little change in the spectral shape. For this reason, in the following, we will refer to PC 1 as the "flaring mode". This component accounts for $68 \%$ of the sample variance. Let us now consider the small spectral fluctuations induced by PC 1 . An increase in luminosity is associated with spectral hardening in the 3-10 keV band, and a slight softening at higher energies which is particularly marked in the $10-30 \mathrm{keV}$ band. This spectral variability suggests a variable power-law spectrum with fixed spectral index moving on top of a constant thermal emission disc emission plus reflection component. Despite these small spectral variations, PC1 correlates very well with the highenergy flux. A least square fit shows that the $3-200 \mathrm{keV}$ flux relates to $\alpha_{1}$ through:

$F_{3-200}=\bar{F}_{3-200}\left(1+7.64 \alpha_{1}\right)$,

with a linear correlation coefficient of 0.98 . So that $\alpha_{1}$ can be viewed as a tracer of the hard X-ray luminosity of the source.

As shown in Fig. 7, the second PCA component (PC 2) can be described roughly as a pivoting of the spectrum around $10 \mathrm{keV}$. The two spectra obtained for the minimum and maximum values of the $\alpha_{2}$ parameter controlling the strength of 
PC 2 are reminiscent of the canonical LHS and HSS spectra. This component is responsible for $27 \%$ of the sample variance, and will be referred as the "pivoting mode". $\alpha_{2}$ can thus be seen as a tracer of the hardness of the high-energy spectrum.

The third PCA component (PC 3) accounts for only $2 \%$ of the sample variance. It is dominated by fluctuations of the relative normalisation of the spectrum in the $J E M-X$ band and ISGRI/SPI energy range. This component is most likely an instrumental artefact due to known calibration issues related to the dithering observation mode. Indeed, since the pointed direction changes between successive science windows and as the effects of vignetting and background non-uniformity are not perfectly corrected in the present release of the data analysis software, this results in spurious variability that affects mainly the relative flux normalisation between instruments. This effect is particularly strong in $J E M-X$ for which systematic errors as large as $30 \%$ are expected for a source 5 degrees off axis. PCA enables us to disentangle such instrumental effects from the intrinsic variability. Because they are independent of the intrinsic source variations and as long as they are not dominant, they are filtered and isolated into higher order PCA components.

Similarly variability due to statistical noise is filtered out into higher order components. From the statistical uncertainties on the measured count rates we expect $3 \%$ of the observed variance to be due to statistical noise. As the first 3 PCA components already account for $97 \%$ of the sample variance, the higher order components are most likely due to noise. Figure 8 shows the contribution of PC 1, PC 2, PC 3 and statistical noise to the observed variance spectrum (i.e. the measured variance as a function of photon energy). These 4 components are enough to account for the observed variance within a few percents at all energies.

Since PC 3 is likely to be an instrumental artefact and higher order components are probably noise, we conclude that the intrinsic source variability is largely dominated by PC 1 (flaring mode) and PC 2 (pivoting mode). Figure 9 shows the time evolution of the PCA parameters $\alpha_{1}$ and $\alpha_{2} . \alpha_{1}$, which traces the changes in bolometric luminosity at nearly constant spectra shows important variability on time scales of order of a few hours or less, but no clear systematic trend during the 4 days of observation. In contrast, $\alpha_{2}$, which roughly traces the hardness of the spectrum, seems to vary on longer time scales: it jumps during the first 2 days then decreases in the second part of the observation. This suggests that the physical mechanisms responsible for PC 1 and PC 2 are distinct (which is also expected from the fact that, by construction, PC 1 and PC 2 are linearly independent) and apparently acting on different time scales.

\subsection{Radio/high-energy correlation}

In order to study the possible correlations between the radio and hard X-ray emission, we selected the science windows for which we had simultaneous radio pointings. When there were several radio pointings falling within a given science window we use the mean radio flux. The resulting light curve is shown in the bottom panel of Fig. 9. A comparison with the time

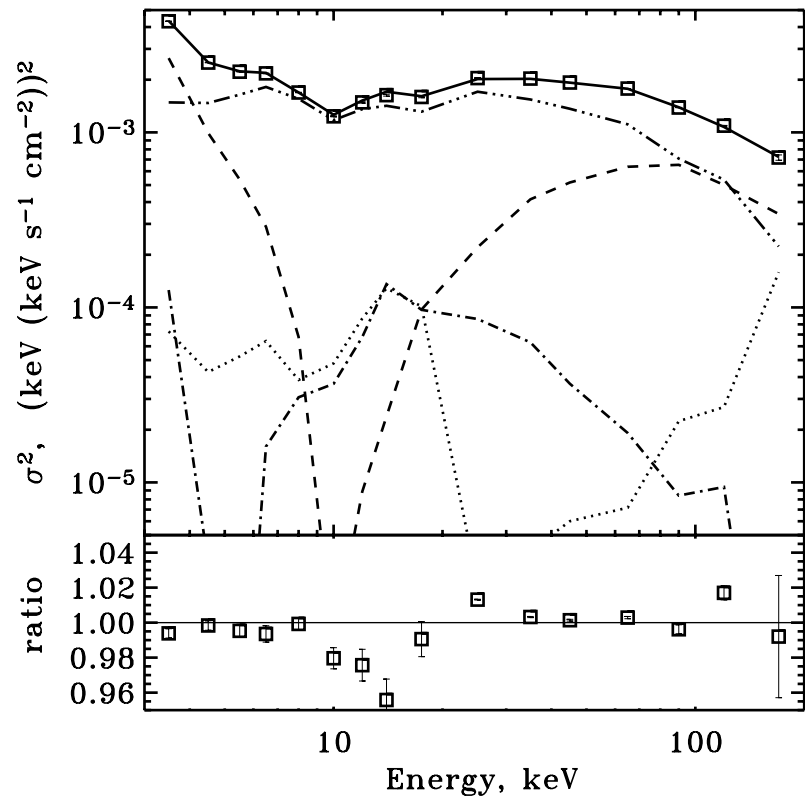

Fig. 8. Observed variance spectrum (squares). The curves show the contribution of PC 1 (3 dots-dash), PC 2 (dashes), PC 3 (dot-dash), estimated statistical noise (dots) and their sum (solid). The bottom panel shows ratio of the contribution of PC $1+\mathrm{PC} 2+\mathrm{PC} 3+$ noise to the total observed variance (values exceeding unity might be due to overestimated statistical noise).

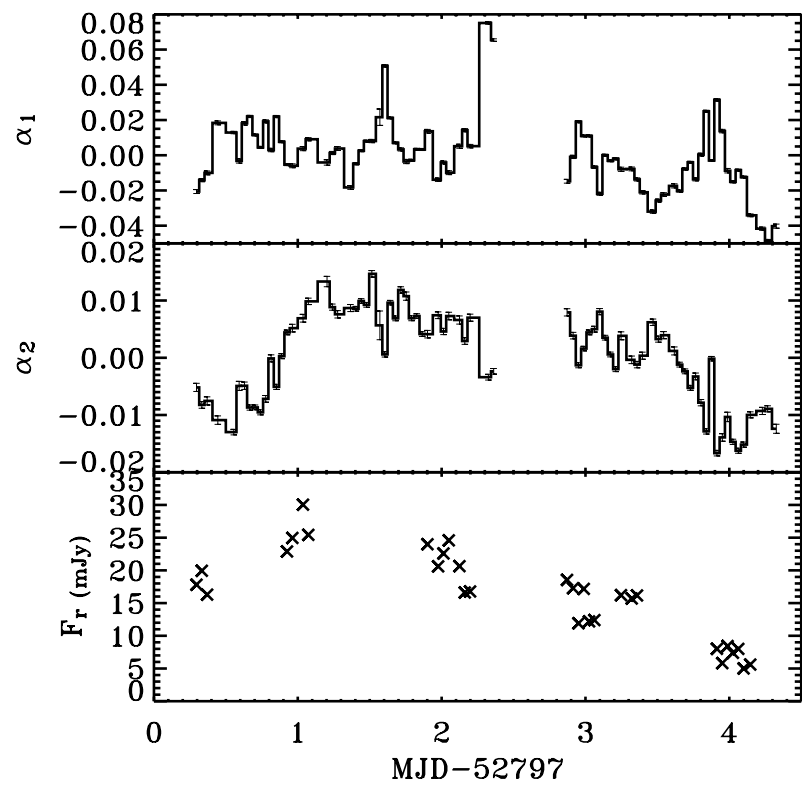

Fig. 9. Evolution of the parameters associated to PC 1 (top), PC 2 (middle) and radio light curve (bottom) during the observation.

evolution of $\alpha_{1}$ and $\alpha_{2}$ indicates that the radio flux tends to follow the evolution of the pivoting mode. In other words, the radio flux tends to be stronger when the hard X-ray spectrum is harder. This is clearly seen in Fig. 10 which shows that the radio emission is strongly correlated to $\alpha_{2}$. The correlation is highly significant. The Spearman rank test correlation coefficient is 0.78 corresponding to a probability that the correlation is by chance of $2 \times 10^{-7}$. On the other hand, there is no hint 

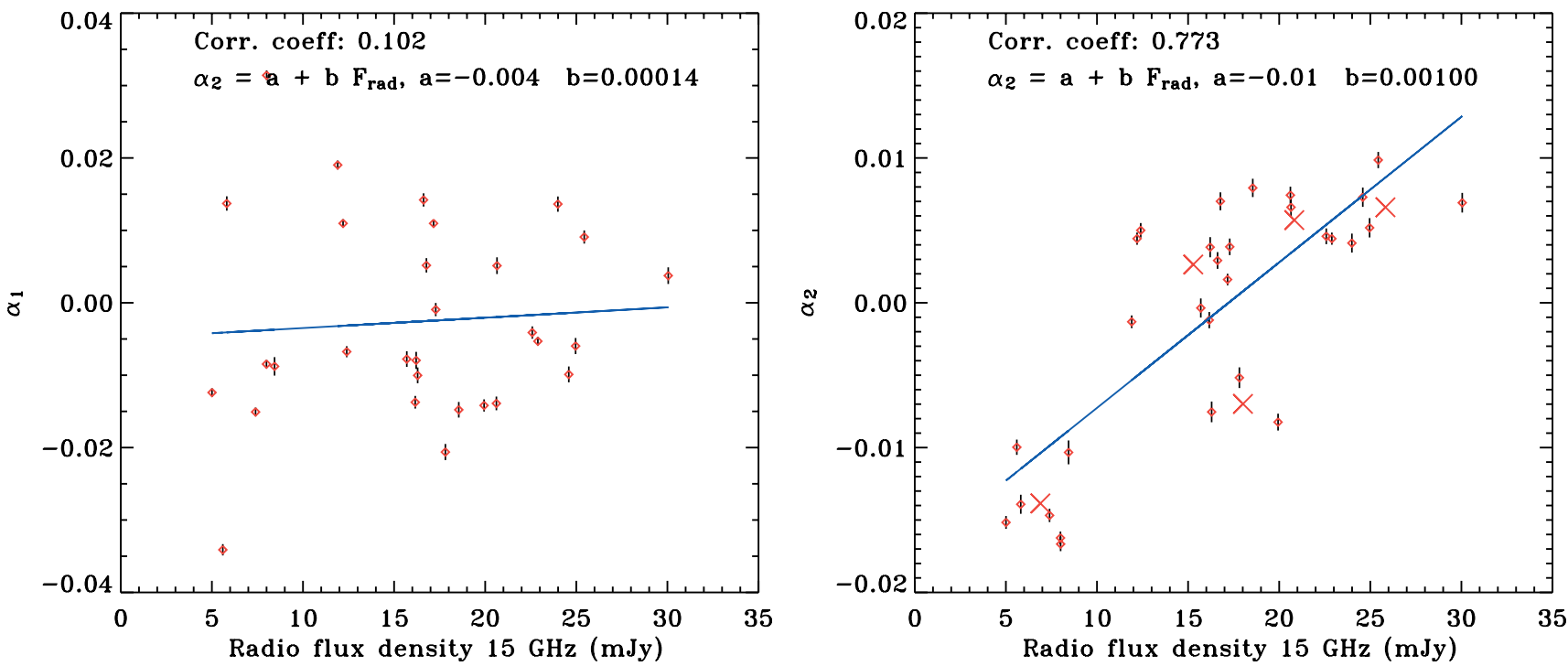

Fig. 10. PCA parameters $\alpha_{1}$ (left panel) and $\alpha_{2}$ (right panel) as a function of the radio flux (diamonds). In both panels, the best linear fits are shown by the solid lines. The crosses indicate the time average over each of the five periods of nearly continuous radio coverage (see Fig. 9). While there is no convincing correlation between the radio flux and $\alpha_{1}$, the radio flux is correlated to $\alpha_{2}$ at highly significant level.

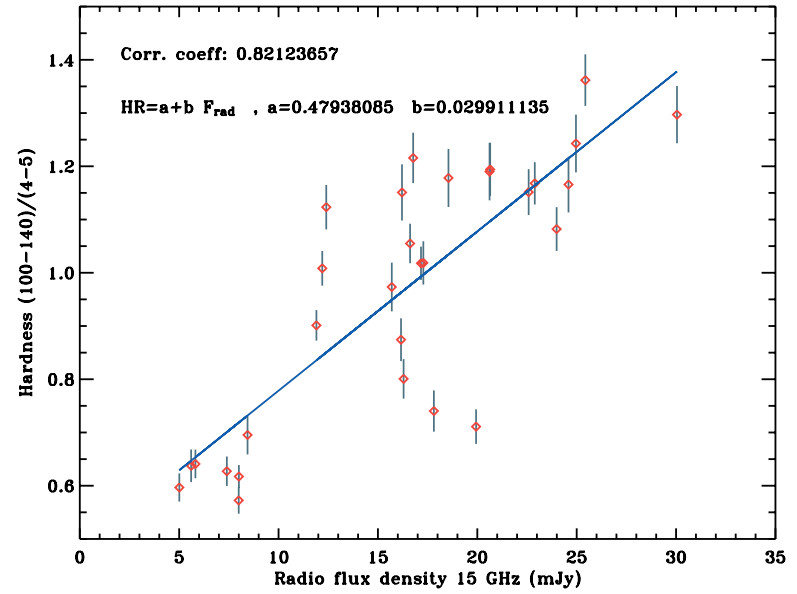

Fig. 11. Correlation between radio flux density and $(100-140 \mathrm{keV}) /(4-$ $5 \mathrm{keV}$ ) hardness.

of a correlation with the flaring mode as can be seen in the left panel of Fig. 10.

It is also worth noting that the correlation between the pivoting mode and the radio flux is valid only when we consider the data over more than one day, we find no evidence for a correlation on shorter time-scales. Therefore, our PCA analysis shows that on time scales of days, the radio jet activity is correlated with hardness of the high-energy spectrum rather than hard X-ray luminosity.

Obviously this can be seen directly from the light curves, although less comprehensively. For instance Fig. 11 shows a strong correlation between the (100-140 keV)/(4-5 keV) hardness and the radio flux. Figure 12 shows that the radio flux tends to be anti-correlated with the X-ray flux (3-7 keV) and correlated with the soft gamma-rays $(>15 \mathrm{keV})$. This dependence of the INTEGRAL/radio flux correlation confirms both the presence of a pivot point located around $10 \mathrm{keV}$ and the correlation of the radio luminosity with the pivoting of the

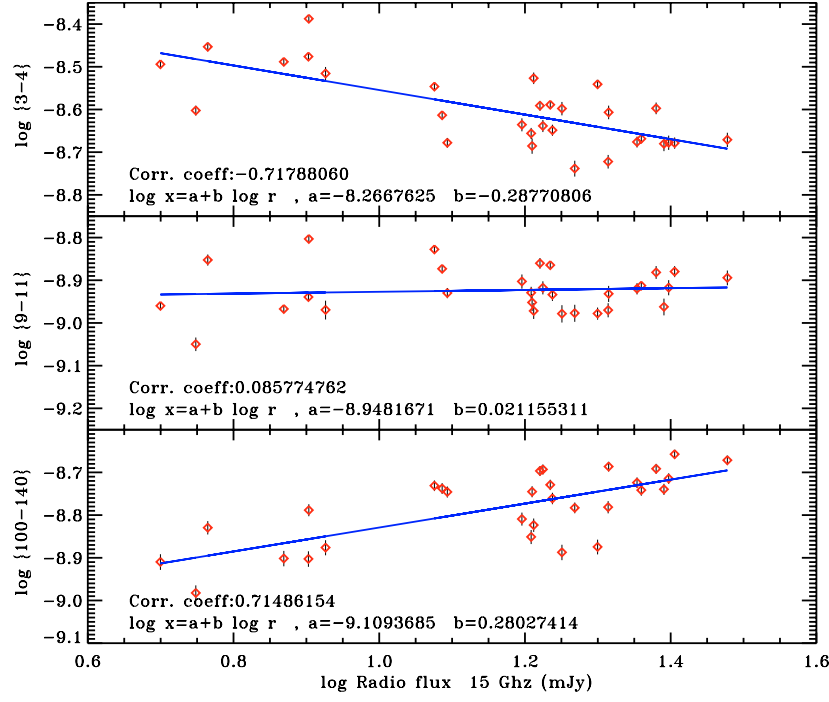

Fig. 12. Flux ( $\left.\mathrm{erg} \mathrm{cm}^{-2} \mathrm{~s}^{-1}\right)$ in different energy bands (as indicated in $\mathrm{keV}$ ), versus $15 \mathrm{GHz}$ radio flux density.

spectrum. However, without PCA it would have been very difficult to demonstrate the presence of the 2 independent variability modes. PCA also brings a much clearer information on the spectral variability due to each mode, and, on top of that, enables one to filter out systematic and statistical errors.

\section{Discussion}

We have shown that, during our IMS observation, the variability of Cygnus X-1 can be described by two independent variability modes:

- On time-scales of a few hours or less there are important changes in luminosity with little spectral variations (flaring mode). 


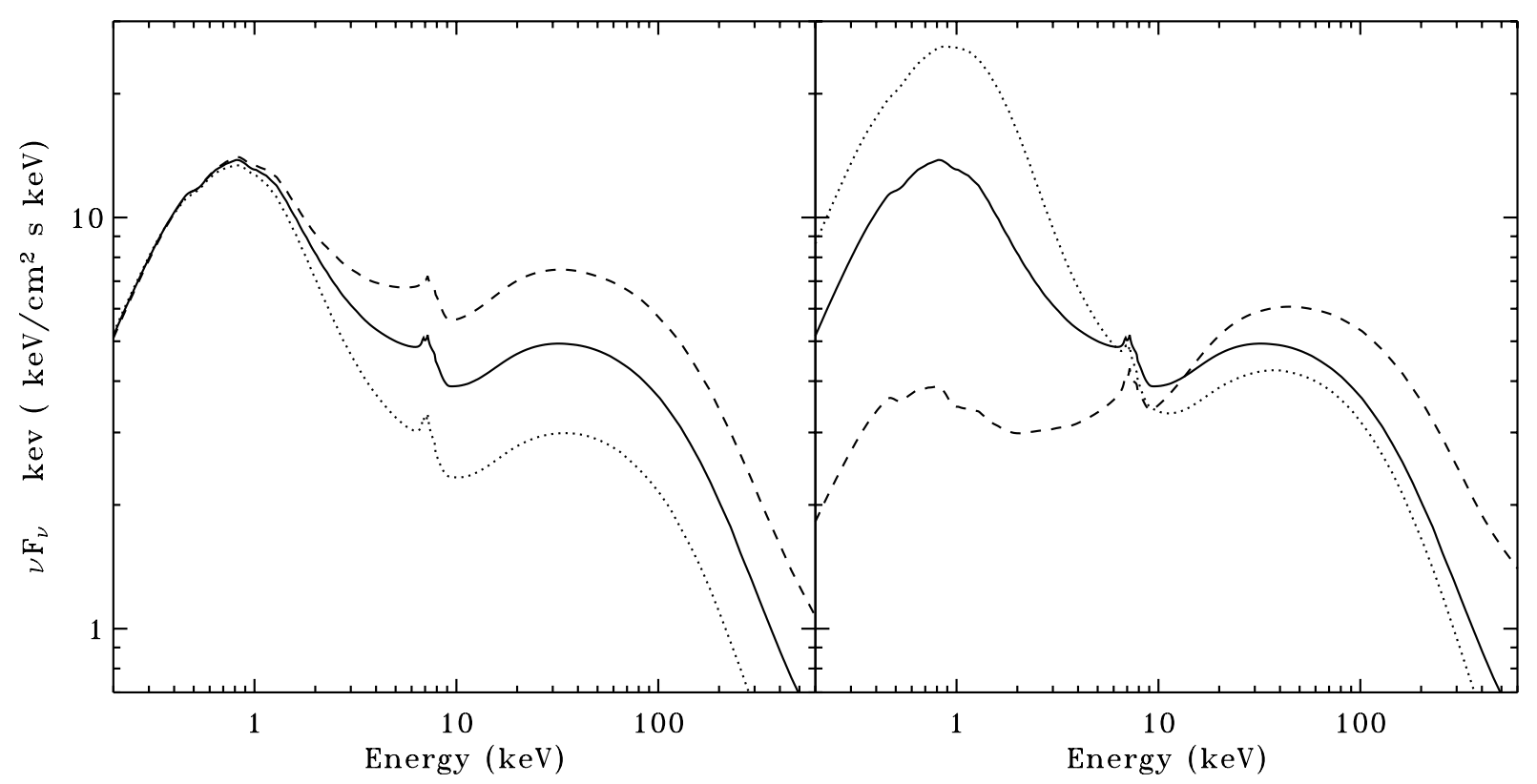

Fig. 13. Left panel: effect of varying $l_{\mathrm{h}}$ by a factor of 2 on the EQPAIR model with monoenergetic injection (see Sect. 2.4). Solid curve: unabsorbed best-fit model $\left(l_{\mathrm{h}}=8.5\right)$; Dotted curve $l_{\mathrm{h}}=5.7$; Dashed curve: $l_{\mathrm{h}}=11.9$. Right panel: effect of varying the soft photons flux by a factor of 8. Solid curve: unabsorbed best-fit model $\left(T_{\text {disc }}=0.3 \mathrm{keV} ; l_{\mathrm{h}} / l_{\mathrm{s}}=0.85\right)$. Dotted curve: $T_{\text {disc }}=0.357 \mathrm{keV}$ and $l_{\mathrm{h}} / l_{\mathrm{s}}=0.42$. Dashed curve: $T_{\mathrm{disc}}=0.212 \mathrm{keV}$ and $l_{\mathrm{h}} / l_{\mathrm{s}}=3.4$.

- On longer time scales there is a spectral evolution with the the spectrum pivoting around $10 \mathrm{keV}$.

We further showed that while there is no hint for a correlation between the radio flux and the flaring mode, the radio is strongly correlated with the pivoting of the spectrum, in the sense that the radio flux is stronger when the hard X-ray spectrum is harder. This result strongly differs from what is usually reported in the LHS. Indeed, the radio flux is then positively correlated with the soft X-ray emission (3-25 keV, Corbel et al. 2000, 2003; Gallo et al. 2003).

However, our results are not in conflict with these observations since Cygnus X-1 was not in a typical LHS but in an IMS, or rather was switching between different IMSs. Indeed, compilations of LHS and HSS spectra suggest that the spectral transition between LHS and HSS occurs through a pivoting around $10 \mathrm{keV}$ (see e.g. Fig. 9 of McConnell et al. 2002). The evolution of the $\alpha_{2}$ parameter shown in Fig. 9 indicates that the source, initially in a "soft" IMS, switched to a harder state during the first 2 days of observation and then transited back toward a "soft" state.

Actually, the transition from LHS to HSS is known to be associated with a quenching of the radio emission (Corbel et al. 2000; Gallo et al. 2003). As the transition to the HSS also corresponds to a strong softening of the spectrum, this is consistent with the correlation between hardness and radio flux: when, during the observation, the source gets closer to the HSS the spectrum softens and simultaneously the radio flux decreases. We also note that a recent analysis of Ryle and RXTE data of Cygnus X-1 (Gleissner et al. 2004) interestingly shows the same correlation tendencies during failed state transitions (Ryle/ RXTE/PCA: moderate anti-correlation,
Ryle/ RXTE/HEXTE: correlation) as reported here, albeit on timescales from weeks to years.

It is interesting to speculate on the cause of the two variability modes. We tried to reproduce such variability modes by varying the parameters of the hybrid thermal/non-thermal Comptonisation models considered in Sect. 2.4. We used the best-fit model obtained with EQPAIR and mono-energetic particle injection. We choose this model because it gives a better fit of the average INTEGRAL spectrum than the power-law injection model, still avoiding the complication of an additional component that cannot be computed self consistently. Actually, similar results are obtained for all of the 3 models we used to fit the broad band spectrum.

As shown in the left panel of Fig. 13 it is possible to produce variations in luminosity by a factor comparable to what is observed and little spectral changes in the INTEGRAL band by varying the coronal compactness $l_{\mathrm{h}}$ by a factor of 2 . In this context the flaring mode would correspond to variations of the dissipation rate in the corona possibly due to magnetic reconnection. This variability mode seems to be a characteristic of the HSS (Zdziarski et al. 2002). As we show here, it also provides a major contribution to the variability of the IMS.

Regarding the pivoting mode, it can be produced by changes in the flux of soft cooling photons at constant dissipation in the hot phase. We performed simulations assuming that the accretion disc radiates like a blackbody i.e. its flux $F_{\text {disc }} \propto l_{\mathrm{s}} \propto T_{\max }^{4}$ and constant $l_{\mathrm{h}}$. For an increase of the disc temperature by a factor of 1.7, the disc luminosity grows by a factor of 8. As in this model, the disc flux also corresponds to the soft cooling photon input in the corona and the heating $\left(\propto l_{\mathrm{h}}\right)$ is kept constant, this leads to a steepening of the spectrum with a pivot around $10 \mathrm{keV}$ of similar amplitude as in PC 2 (see Fig. 13). For the $1996 \mathrm{HSS}, \mathrm{G} 99$ found a ratio $l_{\mathrm{h}} / l_{\mathrm{s}} \sim 0.3$ while 
in the LHS $l_{\mathrm{h}} / l_{\mathrm{S}}$ ranges between 3.5 to 15 (Ibragimov et al. 2005). The range of $l_{\mathrm{h}} / l_{\mathrm{S}}(0.4-3.4)$ required to reproduce the observed amplitude of the pivoting mode matches almost exactly the intermediate range between the HSS and the lower limit of the LHS. The source initially in a (quasi) HSS evolved toward the LHS but as soon as it was reached, it went back toward the HSS.

Since, in the INTEGRAL band, the constraints on disc thermal emission are loose we did not attempt to model the data with a varying inner disc radius which is, moreover, difficult to disentangle from fluctuations of the disc temperature. In the fitted models as well as the models shown in Fig. 13, the inner disc radius is fixed at $6 R_{\mathrm{g}}$. Nonetheless our result would also be consistent with the disc moving inward and outward of the hot phase during the state transitions. Indeed, when the inner disc radius is approaching the black hole, its maximum temperature and luminosity increases ${ }^{3}$ leading to a more efficient cooling of the hot flow/corona. The anti-correlation between radio flux and disc luminosity would be due to the jet expanding when the cold accretion disc recedes and then shrinking in the second phase of the observation when the disc moves back inward. It is interesting to note that the change in disc flux required (a factor of $\sim 8$ ) to explain the spectral evolution is comparable to the amplitude of the variations of the radio flux (a factor of $\sim 6$ ). This suggests a direct relation between the disc flux and jet power. The overall change in bolometric luminosity occuring during the PC2 transition estimated from the fiducial "hard" and "soft" state models shown on the left panel of Fig. 13, is about a factor of 2 . Because of the relatively short time scale ( a day) on which the variation in luminosity occurs, it is unlikely to be driven by changes in the mass accretion rate. Most probably, it is due to a change in the radiative efficiency of the flow. The accretion flow could be less efficient in the LHS, because about half of the accretion power is either swallowed by the black hole or pumped into the jet, while, in the HSS, the cold disc is expected to be radiatively efficient.

The contribution of the jet power to the total energetic output of black hole candidates in the LHS is a matter of debate. It has been argued that the jet could be strong and even dominant (Fender et al. 2003; Malzac et al. 2004). The jump in luminosity by a factor of 2 that we infer during our mini state transition sets an upper limit to the jet power, namely, the jet power at a luminosity just below the transition is at most comparable to the X-ray luminosity. This uppper limit is in aggreement with a recent study of the jet interaction with the surrounding interstellar medium by Gallo et al. (2005). According to these authors, the jet power in Cygnus X-1 represents between 6 to $100 \%$ of the X-ray luminosity at the peak of the LHS. In our model, the upper limit is reached if there is no advection into the black hole. In this case the mini-transition pivoting mode would correspond to a redistribution of the accretion power between the jet and the cold accretion disc. In this case all LHS sources would be jet dominated, since, according to the jet and X-ray power scaling laws of Fender et al. (2003), the jet share

\footnotetext{
${ }^{3}$ Unless the mass accretion rate is reduced by a larger amount, which seems very unlikely, the evidence being rather that the accretion rate is often (but not always) larger in the soft than in the LHS.
}

of the energy budget is increasingly larger at lower luminosities. However, the amplitude of the luminosity changes we infer during the mini-transition (i.e. the factor of 2) is model dependent and also depends on the strength of the thermal disc contribution to the time-averaged spectrum, which is poorly constrained with the INTEGRAL data. Similar observations with and even broader spectral coverage (including soft X-rays) are required to consolidate this result.

Moreover, although our data point toward an inefficient accretion flow in the LHS, it does not tell us about the cause of this inefficiency: pure advection as well as jet dominated accretion flows are both viable possibilities. We note that a similar conclusion was reached by Chaty et al. (2003), on the basis of the analysis of the multi-wavelength spectrum of the transient black hole binary XTE J1118+480.

The evolution of the hard X-ray corona luminosity during our IMS observation is very puzzling. Indeed, if, as commonly believed for the LHS, the corona constitutes the base of the jet, it is difficult to conceive that changes in the jet power and/or extension is not associated to changes in the energetics of the corona. The apparent lack of response of the radio jet to the (short time-scale) X-ray fluctuations could well be due to the time delays required to propagate the information from the corona to the distant radio emitting region, which moreover have very different sizes. However, one would still expect the corona/hot accretion flow to track the longer timescale evolution of the radio jet and also respond to changes in the disc power and/or distance of the truncation radius. Instead, we infer dramatic changes in the jet and disc power that are anti-correlated with each other, but completely unrelated to the fluctuations of the coronal power. On the one hand, the spectral pivoting described by PC 2 is understood in terms of changes in the disc luminosity at constant coronal luminosity. And, on the other hand, the rapid fluctuations of the coronal power that we do observe through PC 1 are apparently not associated to fluctuations of the cold disc or jet emission. The nature of the instabilities responsible for a coronal activity that is so uncoupled to the jet and disc emission remains to be clarified. In any case, these results indicate that, in the IMS, the corona does not play the same role as in the LHS, and the whole disc-corona-jet interaction seem to work differently.

\section{Conclusion}

The INTEGRAL IMS spectrum of Cygnus X-1 shows a highenergy cut-off or break around $100 \mathrm{keV}$. The shape of this cut-off differs from pure thermal Comptonisation, suggesting the presence of a non-thermal component at higher energies. The average broad band spectrum is well fitted with hybrid thermal/non-thermal Comptonisation models, although some important parameters such as the fraction of non-thermal power are not well constrained because they depend on the assumptions of the model (mono-energetic versus power-law injection, presence or absence of an additional soft component). Models with mono-energetic injection, or models with an additional soft component seem to be favoured over standard power-law acceleration models. 
During our observation, the source presented a strong flux spectral variability occurring through 2 independent variability modes:

i) changes in the dissipation rate in the corona, due to local instabilities or flares, producing a variability of the hard $\mathrm{X}$-ray luminosity on time-scales of hours and no strong spectral alterations. Strikingly, this coronal activity seems to be unrelated to the evolution of the jet and cold disc luminosity.

ii) A slower 4-day evolution starting from a spectrum close to the canonical HSS toward an almost LHS and back. This spectral evolution was characterized by a pivoting of the spectrum around $10 \mathrm{keV}$. It was correlated with the radio emission which was stronger when the hard X-ray spectrum was harder. It is interpreted in terms of a variable soft cooling photon flux in the corona associated with changes in the thermal disc luminosity and radio-jet power. This interpretation suggests a jump in bolometric luminosity of about a factor of 2 during the transition from LHS to HSS, which indicates that the LHS accretion flow is radiatively inefficient, half of the accretion power being possibly advected into the black hole and/or the radio jet.

In the IMS, the jet power appears to be anti-correlated with the cold accretion disc luminosity, while the coronal power fluctuates independently. Apparently, the coupling between accretion and ejection processes differs from that of the LHS where the radio jet and the $\mathrm{X}$-ray corona appear intrinsically linked.

Acknowledgements. This paper is based on observations with INTEGRAL, an ESA project with instruments and science data centre funded by ESA member states (especially the PI countries: Denmark, France, Germany, Italy, Switzerland, Spain), Czech Republic and Poland, and with the participation of Russia and the USA. The Ryle Telescope is supported by PPARC. JM acknowledges financial support from the MURST (COFIN98-02-15-41), PPARC, CNRS and European Union (contract number ERBFMRX-CT98-0195, TMR network "Accretion onto black holes, compact stars and protostars"). This research was also supported in part by the National Science Foundation under Grant No. PHY99-07949.

\section{References}

Bazzano, A., Bird, A. J., Capitanio, F., et al. 2003, A\&A, 411, L389 Bisnovatyi-Kogan, G. S., \& Blinnikov, S. I. 1976, Soviet Astronomy Letters, 2, 191

Belloni, T., Mendez, M., van der Klis, M., et al. 1996, ApJ, 472, L107 Beloborodov, A. M. 1999, ApJ, 510, L123

Bouchet, L., Jourdain, E., Roques, J. P., et al. 2003, A\&A, 411, L377

Bowyer, S., Byram, E. T., Chubb, T. A., \& Friedman, M. 1965, Sci., 147,394

Brocksopp, C., Fender, R. P., Larionov, V., et al. 1999, MNRAS, 309, 1063

Cadolle Bel, M., Sizun, P., Goldwurm, A., et al. 2006, A\&A, 446, 591 (CB05)

Chaty, S., Haswell, C. A., Malzac, J., et al. 2003, MNRAS, 346, 689

Coppi, P. S. 1999, High Energy Processes in Accreting Black Holes, ASP Conf. Ser., 161, 375
Corbel, S., Fender, R. P., Tzioumis, A. K., et al. 2000, A\&A, 359, 251

Corbel, S., Nowak, M. A., Fender, R. P., Tzioumis, A. K., \& Markoff, S. 2003, A\&A, 400, 1007

Di Salvo, T., Done, C., Życki, P. T., Burderi, L., \& Robba, N. R. 2001, ApJ, 547, 1024

Fender, R., Corbel, S., Tzioumis, T., et al. 1999, ApJ, 519, L165

Fender, R. P. 2001, MNRAS, 322, 31

Fender, R. P., Gallo, E., \& Jonker, P. G. 2003, MNRAS, 343, L99

Ferreira, J., Petrucci, P. O., Henri, G., Saugé, L., \& Pelletier, G. 2006, A\&A, 447, 813

Francis P. J., \& Wills B. J. 1999, in Quasars and Cosmology, ed. G. Ferland, \& J. Baldwin, ASP Conf. Ser., 162, 363 [arXiv:astro-ph/9905079]

Frontera, F., Palazzi, E., Zdziarski, A. A., et al. 2001, ApJ, 546, 1027 (F01)

Gallo, E., Fender, R. P., \& Pooley, G. G. 2003, MNRAS, 344, 60

Gallo, E., Fender, R., Kaiser, C., et al. 2005, Nature, 436, 819

Gierliński, M., Zdziarski, A. A., Done, C., et al. 1997, MNRAS, 288, 958

Gierliński, M., Zdziarski, A. A., Poutanen, J., et al. 1999, MNRAS, 309, 496 (G99)

Gleissner, T., Wilms, J., Pottschmidt, K., et al. 2004, A\&A, 414, 1091

Goldwurm, A., David, P., Foschini, L., et al. 2003, A\&A, 411, L223

Haardt, F., \& Maraschi, L. 1993, ApJ, 413, 507

Ibragimov, A., Poutanen, J., Gilfanov, M., Zdziarski, A. A., \& Shrader, C. R. 2005, MNRAS, 362, 1435

Kendall, M. G. 1980, Multivariate Analysis, Second Edition (Charles Griffin and Co. London)

Malzac, J., Beloborodov, A. M., \& Poutanen, J. 2001, MNRAS, 326, 417

Malzac, J., Merloni, A., \& Fabian, A. C. 2004, MNRAS, 351, 253

Markoff, S., Nowak, M. A., \& Wilms, J. 2005, ApJ, 635, 1203

Mittaz, J. P. D., Penston, M. V., \& Snijders, M. A. J. 1990, MNRAS, 242,370

McConnell, M. L., Ryan, J. M., Collmar, W., et al. 2000, ApJ, 543, 928

McConnell, M. L., Zdziarski, A. A., Bennett, K., et al. 2002, ApJ, 572, 984

Mendez, M., \& van der Klis, M. 1997, ApJ, 479, 926

Merloni, A., \& Fabian, A. C. 2002, MNRAS, 332, 165

Narayan, R., \& Yi, I. 1994, ApJ, 428, L13

Pottschmidt, K., Wilms, J., Chernyakova, M., et al. 2003, A\&A, 411, L383

Poutanen, J., \& Svensson, R. 1996, ApJ, 470, 249

Shakura, N. I., \& Sunyaev, R. A. 1973, A\&A, 24, 337

Shapiro, S. L., Lightman, A. P., \& Eardley, D. M. 1976, ApJ, 204, 187

Skinner, G., \& Connell, P. 2003, A\&A, 411, L123

Stirling, A. M., Spencer, R. E., de la Force, C. J., et al. 2001, MNRAS, 327,1273

Vaughan, S., \& Fabian, A. C. 2004, MNRAS, 348, 1415

Westergaard, N. J., Kretschmar, P., Oxborrow, C. A., et al. 2003, A\&A, 411, L257

Winkler, C., Courvoisier, T. J.-L., Di Cocco, G., et al. 2003, A\&A, 411, L1

Zdziarski, A. A., Poutanen, J., Paciesas, W. S., \& Wen, L. 2002, ApJ, 578,357

Zdziarski, A. A., Gierliński, M., Mikołajewska, J., et al. 2004, MNRAS, 351, 791

Życki, P. T., Done, C., \& Smith, D. A. 2001, MNRAS, 326, 1367 